\title{
Advances in chemical modifications of polylactide biodegradable materials
}

\author{
Nan $\mathrm{Li}^{1,}$, and Jiansheng Chen ${ }^{1, b}$ \\ ${ }^{1}$ Corporate Research Center, ABB (China) Limited, Beijing, 100015, P. R. China \\ ${ }^{a}$ nan.li@cn.abb.com, ${ }^{b}$ jiansheng.chen@cn.abb.com,
}

\begin{abstract}
Keywords: Polylactide, Poly (lactic acid), Biodegradable materials, Copolymerization, Chemical modifications.

Abstract. Biodegradable materials have been actively studied in applications of food packaging, agricultural film, and biomedical materials. As one of the most important biodegradable materials, polylactide (PLA) has attracted great attention due to its excellent biodegradable property. Despite favorable performance, PLA has some drawbacks to limit its applications. Various modifications have been applied to improve on the overall properties of PLA. In this paper, a summary on literature review of chemical modifications of PLA-based materials is addressed.
\end{abstract}

\section{Introduction}

Polylactide (PLA) is one of the most important biomedical materials owing to its favorable biocompatible and biodegradable performance [1]. It is known that PLA has been widely applied in biological medicine areas, such as drug controlled release, surgical absorbable suture, and clinical orthopedic fixed materials among others [2-3]. On the other hand, PLA has some drawbacks that limit its applications in biomedical applications. Firstly, PLA has weak hydrophility and thermostability, which hinder its biocompatibility with other materials. Secondly, PLA has high brittleness and weak impact resistance and is difficult to meet the requirements of mechanical strength. Thirdly, the crystallization of PLA is so slow that it greatly influences on the processing properties. Last but not the least, the high manufacturing cost brings a large number of restrictions to the applications of PLA.

In recent years, the modifications of PLA have received considerable attention. A variety of chemical and physical modifications approaches have been employed to increase the overall performance of PLA-based materials [4-8]. In following sections, a review on the chemical modifications of PLA such as copolymerization modifications, surface modifications, and compound modifications are presented.

\section{Copolymerization modifications}

Nowadays, it becomes more and more difficult for PLA homopolymer to meet the requirements of constantly increasing application areas. Hence the copolymerization of lactic acid with other monomers has aroused tremendous research interest $[9,10]$. By adjusting the molecular weight of PLA copolymers, and the type and quantity of comonomers, various functional groups can be introduced to PLA molecular chains, and the properties such as crystallinity, hydrophility, and degradation rate can be greatly enhanced accordingly.

Block copolymerization. Chen et al [11] have reported a new biodegradable PLA-based triblock copolymer. By using ring-opening copolymerization, they successfully prepared poly (D,L-lactide-co-p-dioxanone)-poly (ethylene glycol)-poly (D,L-lactide-co-p-dioxanone) [P (LA-PDO)-PEG-P (LA-PDO)] triblock copolymer. The copolymer showed a typical thermogelling property, and underwent sol-gel and gel-sol transitions with temperature rise from 0 to $50 \square{ }^{\circ} \mathrm{C}$ in aqueous solutions. In this study, the introduction of PDO units into the hydrophobic block had greatly improved on the hydrophility of the copolymers and facilitated the drug formulation. The developed triblock copolymer has demonstrated an ideal material used for drug delivery depot and tissue engineering scaffold. 
Recently, a series of multibranched pentablock copolymers ( $m$ Br5BlC), poly (L-lactide)- $b$-HBP- $b$-poly (ethylene glycol)- $b$-HBP- $b$-poly (L-lactide), were synthesized by Kim et al [12]. By virtue of the ROP methodology, researchers achieved a series of copolymers with the MW range of 4360-15,300 and narrow PDI. The $m$ Br5B1Cs exhibited good biocompatibility as biomaterials for various applications in biomedical areas. Furthermore, the $m \mathrm{Br} 5 \mathrm{BlCs}$ showed good controllability in self-assembly into spherical micelles with an average diameter range of 59-140 nm. These micellar structures possessed no acute and intrinsic cytotoxicity against normal cells, and provided a convenient strategy for drug loading.

Venkatraman et al [13] have recently synthesized a PLA-based four-armed star copolymer, and studied its elastomeric behavior. The star copolymer had a fixed core composition comprising caprolactone (CL) and L-lactide (LA), and an elastomer was obtained by increasing the PLA end block lengths. The elastomeric behavior was studied by using cyclic and creep test. The star copolymer demonstrated that at least $75 \%$ of recovery was achieved. The degradation study of star copolymer was also performed by the researchers. The experimental results indicated that the degradation first occurred in the caprolactone-co-lactide (CLLA) core, followed by the degradation of segments in the PLA ends.

Graft copolymerization. Fares et al [14] have reported biodegradable amphiphiles comprising PLA-based graft copolymers. They prepared 2-hydroxylethyl methacrylate- $c o-N$-vinylpyrrolidone copolymers, (HEMA-co-NVP), and then grafted PLA onto (HEMA-co-NVP) copolymers. The grafted copolymers (i.e., amphiphiles) showed a morphology of porous layers with crispy structure. The sustained release studies of the grafted copolymers was performed. At lower content of PLA moiety, the copolymers showed non-Fickian diffusion release rate, while Fickian diffusion release rate at higher content of PLA was observed. The increase of PLA content in the copolymer increased the drug-sustainability, mainly due to the porous amphiphilic structures that allow controllable release of drug in time interval.

Lee et al [15] have prepared cellulose-graft-poly (l-lactide) (Cell-g-PLLA) copolymer in ionic liquid (IL)/dimethyl sulfoxide (DMSO) co-solvent. The copolymer had higher substitution efficiency at relatively low concentration of l-lactide. The maximum values of molar substitution, degree of lactyl substitution, and polymerization degree of PLLA in the copolymer were 3.76, 1.74, and 2.16, respectively. The prepared cell- $g$-PLLA copolymers showed thermal plasticization with a glass transition temperature of $155^{\circ} \mathrm{C}$. On the other hand, the thermal processibility increased with the increase of the amount of grafted PLLA in the copolymer.

Recently, Geschwind et al [16] have synthesized comb and hyperbranched copolymers based on functional poly (ethylene glycol) and poly (l-lactide). By using a combination of anionic and ring-opening polymerization, they obtained molecular weights in the range of 2000-90 $000 \mathrm{~g} \mathrm{~mol}^{-1}$ and moderate molecular weight distributions $\left(M_{\mathrm{w}} / M_{\mathrm{n}}=1.08-1.37\right)$. The stereocomplex formation of the comb and hyperbranched copolymers was identified by a series of characterization methodologies. Results indicated that comb and hyperbranched copolymers possessed an improved hydrophility property.

\section{Surface modifications}

Surface modification is an effective chemical approach utilized to improve on the surface properties (e.g. hydrophility) of PLA-based materials. A lot of methods have been employed to modify the surface properties of PLA, such as plasma treatment, electron beam radiation, and self-assembly method among others [17].

Punyodom et al [18] have recently carried out a study of the surface modification on poly [(1-lactide)-co-( $\square$-caprolactone)] (PLCL) and poly [(1-lactide)-co-glycolide] (PLGA) copolymers, which are widely used in neural guide tissue regeneration. They used plasma treatment to increase the surface hydrophilicity of the copolymers. It was observed that the attachment and proliferation of ensheathing cells on treated membranes increased by 26 and 32\%, respectively, compared to the 
untreated PLCL and PLGA counterparts. As a result, the plasma treatment on the surface of the polymers enhanced both cell viability $(>95 \%)$ and healthy growth without incurring cytotoxic effects.

Cairns et al [19] have demonstrated the ability of electron beam (e-beam) technology to improve biofunctionality through the manipulation of PLLA surface properties. They treated PLLA samples by using e-beam with varying beam energies and doses in air, and characterized the treated surface of PLLA. Results showed a significant increase in surface wettability after e-beam treatment, and an introduction of oxygen-containing functional groups to PLLA surface was observed. It was also observed that e-beam led to chain scission in the near surface region of PLLA, but it did not affect the surface roughness of PLLA.

Zhu et al [20] have demonstrated new biomacromolecule coatings on PLA based on electrostatic self-assembly technique. They employed poly (ethylene imine) (PEI) to engineer poly (dl-lactide) (PDL-LA) substrate to obtain a stable positively charged surface. Then an extracellular matrix(ECM-) like biomacromolecule, gelatin, was self-assembled onto PDL-LA substrate. The multilayer modified PDL-LA substrate was investigated. Results indicated that the multilayer modified PDL-LA substrate promoted chondrocyte cell attachment and growth. Furthermore, polyelectrolyte self-assembled multiplayers have proven easy and stable to prepare compared with conventional coating methods. The authors believed it would be a promising modification approach for 3-D scaffolds used in drug delivery and tissue engineering.

\section{Compound modifications}

The compound modifications have promoted versatile molecular designs and boosted performance improvement of PLA-based materials [21]. Feijen et al [22] has reported the blends of biodegradable triblock copolymers based on 1,3-trimethylene carbonate (TMC) and different lactides (i.e. L-lactide (LLA), D-lactide (DLA). In the blends of poly (LLA-TMC-LLA) and poly (DLA-TMC-DLA) triblock copolymers, they observed a stereo-complex formation between the enantiomeric PLA segments. These blends have demonstrated good tensile properties and excellent resistance to creep under static and dynamic loading conditions.

Lei et al [23] reported an investigation of stereocomplexed PLA. The researchers firstly synthesized PDLA-PEG-PDLA tri-block copolymers with different PEG and PDLA segment lengths, and then prepared the blend of linear poly (l-lactide) (PLLA) and PDLA-PEG-PDLA. They examined the crystallization behavior of the blend in detail by DSC, XRD and polarized optical microscopy. The results showed that the crystallization temperature and melting temperature of stereocomplexed-PLA increased with increase of PEG segmental length, and PEG could facilitate the spherulite growth rate. On the other hand, both the PDLA and PEG segmental lengths in the tri-block copolymers affect the crystallinity of stereocomplexed-PLA and the stereocomplexation formation process.

\section{Conclusions}

The modification of PLA homopolymer has aroused tremendous research interests. As an important modification approach, chemical modification has been widely employed to enhance PLA's overall performance. By virtue of various chemical medications, the hydrophility, impact resistance, and biocompatibility of PLA can be significantly improved without influencing its biodegrability performance. Among the different chemical modification methodologies, compound modification has attracted an increasing attention for its effective function on molecular designs of PLA, and is becoming one of the most important research directions. With the rapid development of chemical modifications of PLA, a broader application of PLA-based materials in biological medicine areas can be expected in the near future. 


\section{References}

[1] O. Dechy-Cabaret, B. Martin-Vaca and D. Bourissou: Chem. Rev. Vol. 104 (2004), p. 6147

[2] S. Inkinen, M. Hakkarainen, A. Albertsson, and A. Södergård: Biomacromolecules Vol. 12 (2011), p. 523

[3] M. Hillmyer and W. Tolman: Acc. Chem. Res. Vol. 47 (2014), p. 2390

[4] D. Noga, T. Petrie, A. Kumar, M. Weck, A. García and D. Collard: Biomacromolecules. Vol. 9 (2008), p. 2056

[5] E. Třesohlavá*, Š. Popelka, L. Machová and F. Rypáček: Biomacromolecules Vol. 11 (2010), p. 68

[6] P. Sarazin and B. Favis: Biomacromolecules Vol. 4 (2003), p. 1669

[7] Y. Sun and C. He: ACS Macro Lett Vol. 1 (2012), p. 709

[8] M. Stanford and A. Dove: Macromolecules Vol. 42 (2009), p. 141

[9] J. Yu and Z. Qiu: ACS Appl. Mater. Interfaces Vol. 3 (2011), p. 890

[10]I. Keen, A. Yu, H. Cheng, K. Jack, T. Nicholson, A. Whittaker, and I. Blakey: Langmuir Vol. 28 (2012), p. 15876

[11]R. Chen, J. Hao, C. Xiong and X. Deng: Advanced Engineering Materials Vol. 12 (2010), p. B504 [12]J. Wang, M. Kim, D. Kang, H. Suh and I. Kim: J Polym Sci Part A: Polym Chem Vol. 50 (2012), p. 2553

[13]J. Kong, V. Lipik, M. Abadie, G. Deen and S. Venkatraman: J Biomed Mater Res Part A Vol. 100A (2012), p. 3436

[14] M. Fares, S. Assaf and A. Jaber: J. Appl. Polym. Sci. Vol. 122 (2011), p. 840

[15]S. Lee, H. Lee, S. Jeong, H. Kim, S. Lee and T. Oh: J. Appl. Polym. Sci. Vol. 132 (2015), p. 41331

[16]J. Geschwind, S. Rathi, C. Tonhauser, M. Schömer, S. Hsu, E. Coughlin and H. Frey: Macromolecular Chemistry and Physics Vol. 214 (2013), p. 1434

[17]A. Privalova, E. Markvicheva, Ch. Sevrin, M. Drozdova, C. Kottgen, B. Gilbert, M. Ortiz and Ch. Grandfils: J Biomed Mater Res Part A Vol. 103A (2015), p. 939

[18]P. Techaikool, D. Daranarong, J. Kongsuk, D. Boonyawan, N. Haron, W. Harley, K. Thomson, L. Foster and W. Punyodom: Polymer International Vol. 66 (2017), p. 1640

[19] M. Cairns, G. Dickson, J. Orr, D. Farrar, C. Hardacre, J. Sa, P. Lemoine, M. Mughal and F. Buchanan: J Biomed Mater Res Part A Vol. 100A (2012), p. 2223

[20] Huiguang Zhu, Jian Ji, Qinggang Tan, M. A. Barbosa and Jiacong Shen: Biomacromolecules Vol. 4 (2003), p. 378

[21]T. Ouchi and Y. Ohya: J Polym Sci Part A: Polym Chem Vol. 42 (2004), p. 453

[22] Z. Zhang, D. Grijpma and J. Feijen: Macromolecular Chemistry and Physics Vol. 205 (2004), p. 867

[23] Z. Jing, X. Shi, G. Zhang and R. Lei: Polymer International Vol. 64 (2015), p. 1399 